\title{
Field Quality Measurements and Analysis of the LARP Technology Quadrupole Models
}

\author{
R. Bossert, G. Chlachidze, J. DiMarco, V.V. Kashikhin, M. Lamm, P. Schlabach,, M. Tartaglia, J.C. \\ Tompkins, G.V. Velev, A.V. Zlobin, S. Caspi, P. Ferracin, G.I. Sabbi
}

\begin{abstract}
One of the US-LHC accelerator research program goals is to develop and prove the design and technology of $\mathrm{Nb}_{3} \mathrm{Sn}$ quadrupoles for an upgrade of the LHC Interaction Region (IR) inner triplets. Four 1-m long technology quadrupole models with a $90 \mathrm{~mm}$ bore and field gradient of $200 \mathrm{~T} / \mathrm{m}$ based on similar coils and different mechanical structures have been developed. In this paper, we present the field quality measurements of the first several models performed at room temperature as well as at superfluid helium temperature in a wide field range. The measured field harmonics are compared to the calculated ones. The field quality of $\mathrm{Nb}_{3} \mathrm{Sn}$ quadrupole models is compared with the NbTi quadrupoles recently produced at Fermilab for the first generation LHC IRs.

Index Terms- Magnetic Field Measurements, Super-
\end{abstract} conducting accelerator magnets

\section{INTRODUCTION}

$\mathrm{I}^{\mathrm{N}}$ the past several years, Fermilab and LBNL, as part of the IUS-LHC accelerator program (LARP), have collaborated in a research program with main goal to demonstrate that $\mathrm{Nb}_{3} \mathrm{Sn}$ magnets are a viable alternative for a future high luminosity LHC IR quadrupole upgrade [1]. These quadrupoles aim to substitute the recently built by Fermilab and KEK LQX [2][3] NbTi based LHC IR magnets. They have to provide similar or better operational parameters: more than $205 \mathrm{~T} / \mathrm{m}$ at large aperture of $90 \mathrm{~mm}$ with excellent field quality for smaller beta-star and most important to sustain the high radiation conditions of the IRs. As a first step in this research program, four $1-\mathrm{m} \mathrm{Nb}_{3} \mathrm{Sn}$ technology quadrupole (TQ) models with the same type coils assembled in different supporting structures has been built. Two of them (TQS01-02) were produced by LBNL and cold tested at Fermilab. The other two (TQC01-02) are built by Fermilab and up to now only TQC01 was cold tested.

The TQC and TQS magnets use identical coils but different coil support structures. The TQC coil is supported by stainless steel collars borrowed from MQXB program with slight

Manuscript received August 27, 2007. Work supported by the U.S. Department of Energy.

R. Bossert, G. Chlachidze, J. DiMarco, V.V. Kashikhin, M. Lamm, P. Schlabach,, M. Tartaglia, J.C. Tompkins, G.V. Velev, A.V. Zlobin are with Fermilab, P.O. Box 500, Batavia, IL 60510, USA, (e-mails: velev@fnal.gov; vadim@fnal.gov).

S. Caspi, P. Ferracin, G.I. Sabbi are with the Lawrence Berkeley National Laboratory, Berkeley, CA 94720, USA.

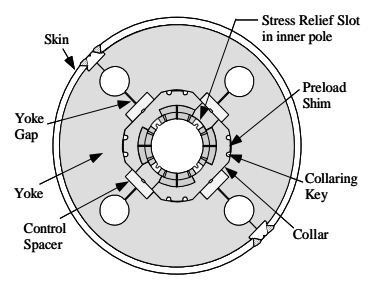

TQC

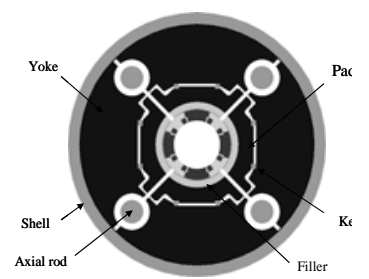

TQS
Fig. 1. Schematic view of the TQC and TQS yoked magnets

modifications [4]. Because of larger Lorentz forces a part of coil prestress is provided by collar interference with the iron yoke. The TQS magnet is based on the bladder and keys technology, where the coil is prestressed by means of the iron pads and thermal shrinkage of outer shell [5].

In this paper, we present the results of magnetic measurements of the two LBL produced TQS and one Fermilab TQC magnets. Room temperature measurements were performed at yoked assembly stages as part of quality control procedures. A set of magnetic measurements were done during cold testing of the magnets.

\section{FIELD MEASUREMENTS}

All results in this paper are expressed in terms of harmonic coefficients defined in a series expansion given by

$$
B_{y}+i B_{x}=B_{2} 10^{-4} \sum_{n=1}^{\infty}\left(b_{n}+i a_{n}\right)\left(\frac{x+i y}{r_{0}}\right)^{n-1}
$$

where $B_{x}$ and $B_{y}$ in (1) are the field components in the Cartesian coordinates, $b_{n}$ and $a_{n}$ are the $2 n$-pole normal and skew coefficients at the reference radius $r_{0}$ of $22.5 \mathrm{~mm}$, chosen at similar fraction of the coil aperture as in MQXB. Probe centering is done using the standard technique of zeroing the dipole component assuming that it is purely generated from a probe offset in the quadrupole field. The right-handed measurement coordinate system is defined with the $\mathrm{z}$-axis at the center of the magnet aperture and pointing from return to lead end.

The magnetic measurements were performed at the Fermilab Vertical Magnet Test Facility. The final set of measurements was performed at the LHC operational temperature (1.9 K superfluid helium) on all the TQ magnets. TQC01 was limited by quench performance and was measured up to $7 \mathrm{kA}$. The field harmonics were measured 
with a rotating coil system that is integrated with a probe translation system. The translation system has ability to position the measurement probe center in the longitudinal direction with a reproducibility of better than $\pm 0.5 \mathrm{~mm}$. A detailed description of the system can be found in [6]-[7]. A new rotational probe with optimal radius of $22 \mathrm{~mm}$, close to the reference one, and length of $100 \mathrm{~mm}$ was built for these measurements. It has a tangential winding for measurement of higher order harmonics as well as specific dipole and quadrupole windings for measurement of the lowest order components of the field [8].

\section{Transfer Function and Geometrical Harmonics}

Fig. 2 shows the measured transfer functions (TF) in the magnets versus the current. The loops are executed with ramp rate of $20 \mathrm{~A} / \mathrm{s}$. Both type of magnets, TQS and TQC, shows distinguishable pattern, which is determined from the iron characteristics. For example, one can see that TQS starts to saturate around $2.0 \mathrm{kA}$ while this effect is not observed in the TQC up to $7 \mathrm{kA}$. We observe $~ 0.5 \%$ discrepancy between the measured and calculated values, which is under investigation. One of the possibilities of this discrepancy is due to a calibration inaccuracy of the measurement probe.

The average geometrical harmonics in the magnet bodies are presented in Table I at $45 \mathrm{~T} / \mathrm{m}$. That gradient was selected above the full penetration field of the superconductor but below the iron saturation in order to reduce the errors associated with these effects. Although achieving a particularly good field quality was not a TQ model target, one can see that harmonics differ from calculations by no more than 4.5 units.

Table II compares the difference between calculations and measurements of TQ magnets with the average harmonics and RMS in the first 3 HGQ and 18 MQXB quadrupole magnets at the half of the coil aperture. One can see that the TQ field harmonics are compatible within the uncertainties with the corresponding averages from HGQ models. The MQXB harmonics are noticeably lower that represents improvement of the NbTi magnet production technology in the HGQ program.

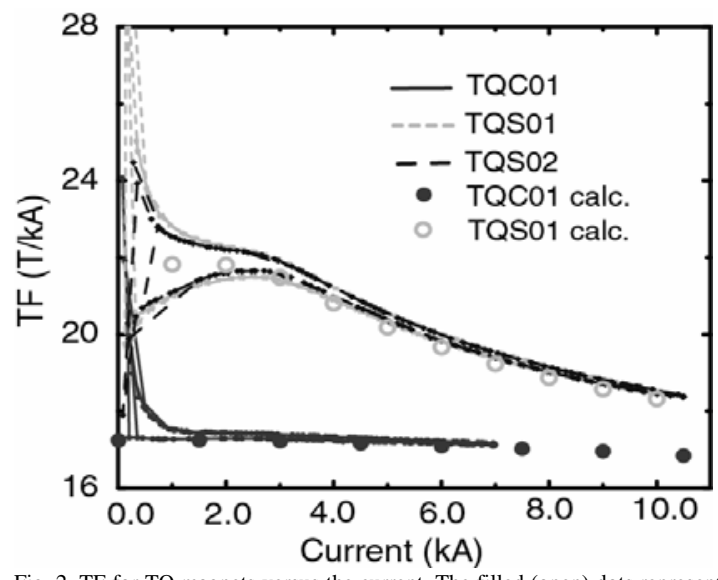

Fig. 2. TF for TQ magnets versus the current. The filled (open) dots represent the calculations for TQC (TQS) respectively.

\begin{tabular}{|c|c|c|c|c|c|c|c|c|}
\hline \multirow{3}{*}{$\mathrm{n}$} & \multirow{2}{*}{\multicolumn{3}{|c|}{ TQC01 }} & \multicolumn{5}{|c|}{ TQS } \\
\hline & & & & \multirow[b]{2}{*}{ calc } & \multicolumn{2}{|c|}{01} & \multicolumn{2}{|c|}{02} \\
\hline & calc & meas & diff & & meas & diff & meas & diff \\
\hline $\mathrm{b}_{3}$ & - & 2.01 & 2.01 & - & -1.46 & -1.46 & 2.98 & 2.98 \\
\hline $\mathrm{b}_{4}$ & - & -1.90 & -1.90 & - & -0.52 & -0.52 & 1.31 & 1.31 \\
\hline$b_{5}$ & - & 0.58 & 0.58 & - & 3.06 & 3.06 & -1.45 & -1.45 \\
\hline$b_{6}$ & 0.90 & 1.71 & 0.82 & 5.00 & 5.40 & 0.40 & 6.23 & 1.23 \\
\hline $\mathrm{b}_{7}$ & - & 0.07 & 0.07 & - & 0.07 & 0.07 & 0.05 & 0.05 \\
\hline $\mathrm{b}_{8}$ & - & 0.01 & 0.01 & - & -0.11 & -0.11 & -0.13 & -0.13 \\
\hline $\mathrm{b}_{9}$ & - & 0.04 & 0.04 & - & 0.02 & 0.02 & 0.10 & 0.10 \\
\hline $\mathrm{b}_{10}$ & 0.00 & -0.06 & -0.06 & -0.04 & 0.02 & 0.06 & -0.05 & -0.01 \\
\hline$a_{3}$ & - & -1.72 & -1.72 & - & 4.41 & 4.41 & 0.66 & 0.66 \\
\hline$a_{4}$ & - & 0.62 & 0.62 & - & -1.99 & -1.99 & 0.82 & 0.82 \\
\hline $\mathrm{a}_{5}$ & - & -1.33 & -1.33 & - & 0.71 & 0.71 & -1.50 & -1.50 \\
\hline $\mathrm{a}_{6}$ & - & -0.10 & -0.10 & - & -0.37 & -0.37 & 0.12 & 0.12 \\
\hline $\mathrm{a}_{7}$ & - & 0.10 & 0.10 & - & -0.11 & -0.11 & -0.01 & -0.01 \\
\hline $\mathrm{a}_{8}$ & - & -0.03 & -0.03 & - & -0.18 & -0.18 & -0.10 & -0.10 \\
\hline $\mathrm{a}_{9}$ & - & 0.08 & 0.08 & - & -0.02 & -0.02 & 0.02 & 0.02 \\
\hline$a_{10}$ & - & 0.00 & 0.00 & - & 0.00 & 0.00 & -0.08 & -0.08 \\
\hline
\end{tabular}

TABLE II COMPARISON OF TQ AND MQXB HARMONICS

\begin{tabular}{rccccc}
\hline \hline & TQC01 & TQS01 & TQS02 & HGQ & MQXB* \\
\hline $\mathrm{b}_{3}$ & 2.01 & -1.46 & 2.98 & $0.20 \pm 0.68$ & $-0.04 \pm 0.59$ \\
$\mathrm{~b}_{4}$ & -1.90 & -0.52 & 1.31 & $0.14 \pm 0.01$ & $0.13 \pm 0.13$ \\
$\mathrm{~b}_{5}$ & 0.58 & 3.06 & -1.45 & $0.60 \pm 0.69$ & $0.00 \pm 0.17$ \\
$\mathrm{~b}_{6}$ & 0.82 & 0.40 & 1.23 & $0.96 \pm 0.40$ & $0.11 \pm 0.29$ \\
$\mathrm{~b}_{7}$ & 0.07 & 0.07 & 0.05 & $-0.02 \pm 0.04$ & $-0.00 \pm 0.04$ \\
$\mathrm{~b}_{8}$ & 0.01 & -0.11 & -0.13 & $0.01 \pm 0.01$ & $-0.01 \pm 0.01$ \\
$\mathrm{~b}_{9}$ & 0.04 & 0.02 & 0.10 & $0.00 \pm 0.01$ & $0.00 \pm 0.01$ \\
$\mathrm{~b}_{10}$ & -0.06 & 0.06 & -0.01 & $-0.02 \pm 0.01$ & $0.02 \pm 0.01$ \\
$\mathrm{a}_{3}$ & -1.72 & 4.41 & 0.66 & $0.19 \pm 0.38$ & $0.01 \pm 1.00$ \\
$\mathrm{a}_{4}$ & 0.62 & -1.99 & 0.82 & $0.51 \pm 0.15$ & $-0.22 \pm 0.40$ \\
$\mathrm{a}_{5}$ & -1.33 & 0.71 & -1.50 & $0.04 \pm 0.19$ & $0.01 \pm 0.18$ \\
$\mathrm{a}_{6}$ & -0.10 & -0.37 & 0.12 & $0.03 \pm 0.03$ & $-0.10 \pm 0.18$ \\
$\mathrm{a}_{7}$ & 0.10 & -0.11 & -0.01 & $-0.01 \pm 0.02$ & $-0.00 \pm 0.03$ \\
$\mathrm{a}_{8}$ & -0.03 & -0.18 & -0.10 & $0.02 \pm 0.03$ & $-0.00 \pm 0.03$ \\
$\mathrm{a}_{9}$ & 0.08 & -0.02 & 0.02 & $-0.00 \pm 0.01$ & $0.00 \pm 0.01$ \\
$\mathrm{a}_{10}$ & 0.00 & 0.00 & -0.08 & $-0.00 \pm 0.03$ & $-0.00 \pm 0.02$ \\
\hline \hline
\end{tabular}

* MQXB harmonics are at $\sim 200 \mathrm{~T} / \mathrm{m}$
Formatted: Bullets and Numbering 


\section{Iron saturation effect}

The iron saturation effect was extracted as an average value between up and down ramps of the measured hysteresis loops at $20 \mathrm{~A} / \mathrm{s}$. Fig. 3-4 show the calculated and measured iron saturation effect in dodecapole for TQC and TQS magnets. The geometrical harmonics were subtracted from the data at $45 \mathrm{~T} / \mathrm{m}$.

The maximum dodecapole deviations are $\sim 0.5$ units in TQC01 magnet and $\sim 3$ units in TQS01-02 in the measured current range. The larger iron saturation effect in TQS magnets is due to the iron pads placed next to the coil. If necessary, it can be corrected by introducing holes into appropriate places in iron pads and/or yoke.

In comparison, the maximum dodecapole deviation due to the iron saturation in MQXB magnets, that have the same iron yoke as TQC, was in the order of $\sim 0.2$ units at the same fraction of the coil aperture.

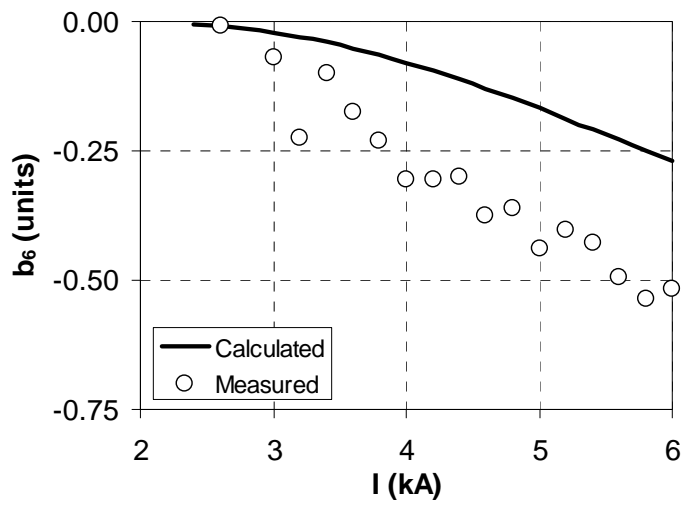

Fig. 3. Iron saturation effect in TQC01.

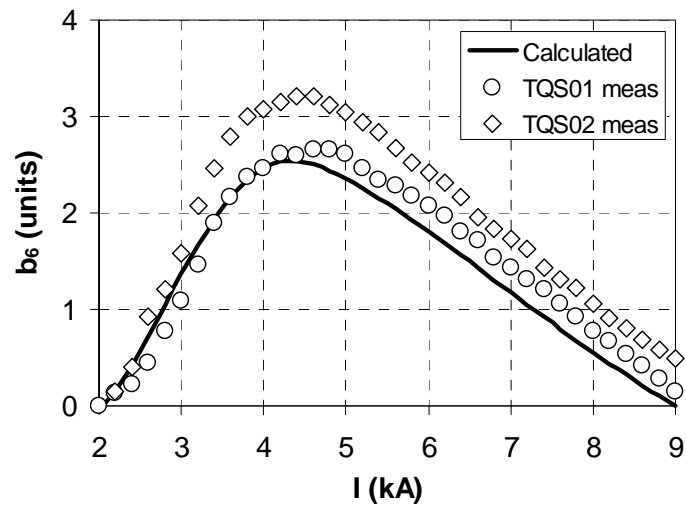

Fig. 4. Iron saturation effect in TQS01-02.

\section{Persistent and eddy current effect}

Current loops at 20, 40 and $80 \mathrm{~A} / \mathrm{s}$ for TQ quadrupoles have been executed. Fig. 5-6 show the measured dodecapole loops of TQC and TQS magnets at $20 \mathrm{~A} / \mathrm{s}$. The TQC01 and TQS01 magnets had identical coils made of the same MJR conductor that resulted in similar coil magnetization effects and dodecapole loop widths. The TQS02 magnet was made from RRP conductor with larger magnetization. However, the Fig. 6 shows about the same loop width for TQS01 and TQS02.

The reason of that becomes clear from the Fig. 7-8, summarizing the widths of transfer function $\Delta \mathrm{TF}=\left(\mathrm{TF}^{\text {up ramp }}\right.$. $\left.\mathrm{TF}^{\text {down ramp }}\right)$ and dodecapole $\Delta \mathrm{b}_{6}=\left(\mathrm{b}_{6}{ }^{\text {up ramp }}-\mathrm{b}_{6}{ }^{\text {down ramp }}\right)$ loops at $45 \mathrm{~T} / \mathrm{m}$ at different ramp rates. One can see that because of larger eddy current effect in TQS02 magnet, the $\Delta \mathrm{b}_{6}$ at $20 \mathrm{~A} / \mathrm{s}$ was reduced to about the same value as in TQS01. Extrapolation of $\Delta b_{6}$ to zero ramp rate clearly shows larger coil magnetization effect in TQS02 magnet.

One can see that all data can be well approximated by straight lines with $\Delta \mathrm{TF}$ extrapolating to nearly the same point at zero ramp rate, indicating small measurement errors with respect to the magnitude of the eddy current effect. The TQC01 have negative slopes of $\Delta \mathrm{TF}$ and $\Delta \mathrm{b}_{6}$ functions, while the TQS01-02 magnet has positive slope in the $\Delta \mathrm{TF}$ and negative slope in $\Delta \mathrm{b}_{6}$. Also, the slope of $\Delta \mathrm{b}_{6}$ is a factor of two larger than in other two magnets. Such behavior is likely due to different interstrand contact resistances in TQ models caused by different mechanical prestresses and conductors.

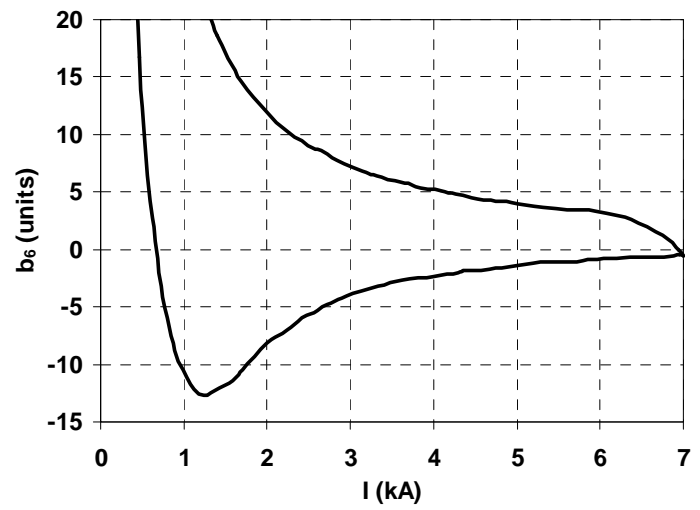

Fig. 5. Normal dodecapole hysteresis for TQC01 at ramp rate of $20 \mathrm{~A} / \mathrm{s}$.

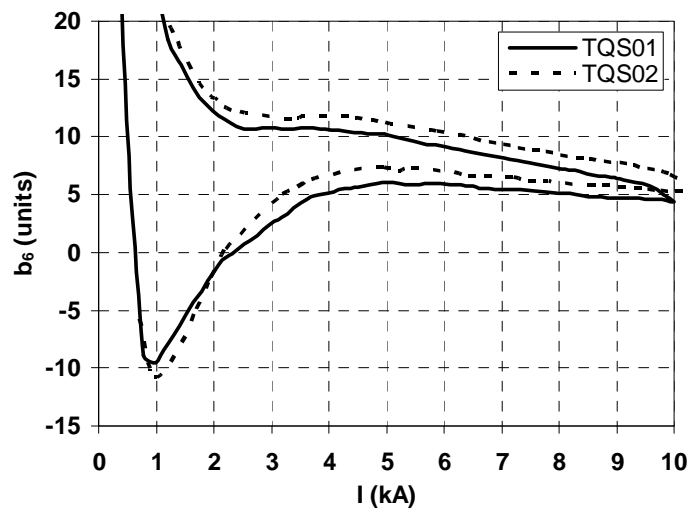

Fig. 6. Normal dodecapole hysteresis for TQS01-02 at ramp rates of $20 \mathrm{~A} / \mathrm{s}$. 


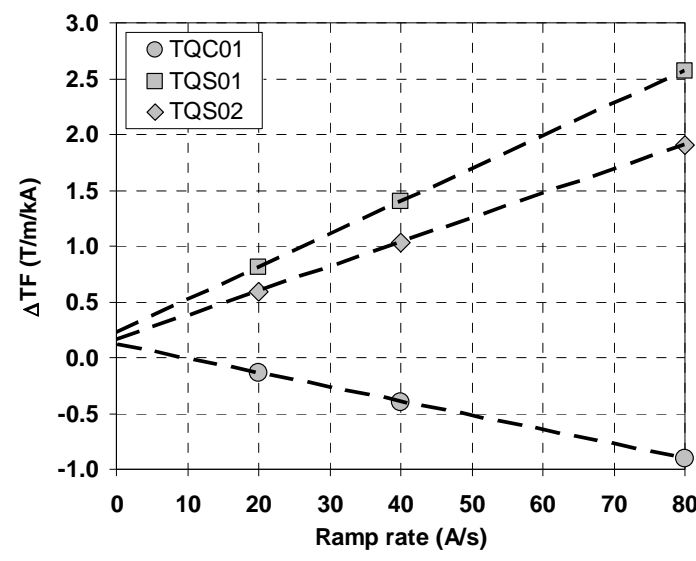

Fig. 7. Width of the transfer function loop as function of the ramp rate.

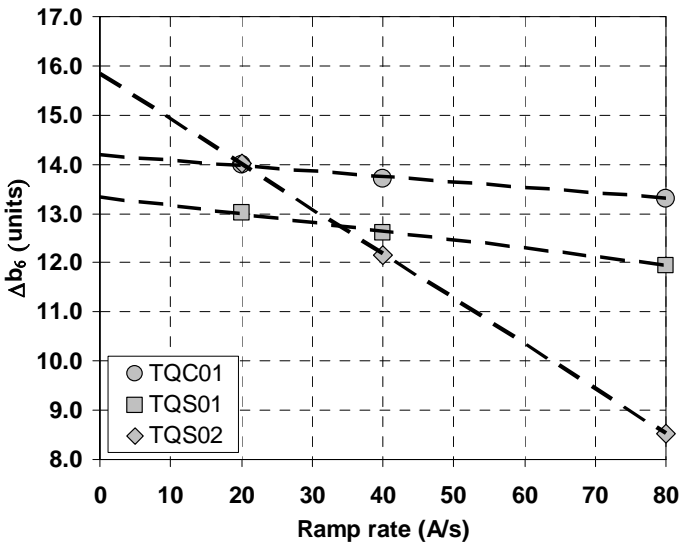

Fig. 8. Width of the dodecapole loop as function of the ramp rate.

\section{Long-term Dynamic Effects}

Long-term dynamic effects in superconducting magnets play an important role in the operation of modern accelerators. This well-known phenomenon is usually associated with the decay and subsequent snapback of the allowed field components at injection [9], [10].

To investigate these effects in the TQ quadrupoles, we performed measurements with an accelerator current profile similar to the one used for the LHC IR quadrupole tests. The I important characteristic of this profile is the duration of the injection plateau, which was set to $\sim 900$ s. In our measurements, we focused on the decay and snapback in normal dodecapole component, the first allowed multipole.

The decay and snapback was not observed in TQ magnets | (Fig. 9), while the average amplitude in the MQXB NbTi quadrupoles was found to be $0.39 \pm 0.11$ [2]. The long-term decay and snap-back was not observed also in other $\mathrm{Nb}_{3} \mathrm{Sn}$ magnets made of similar conductors.

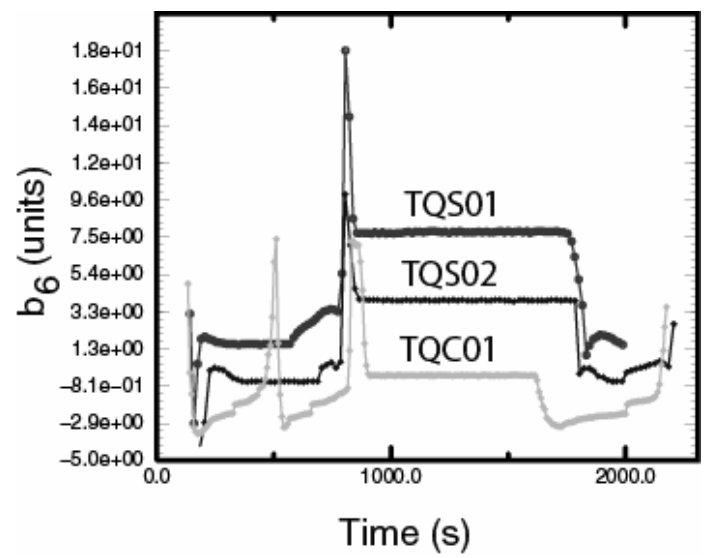

Fig. 9. Examples of the typical profiles for measurement of the decay and snapback of the dodecapole component for a duration of injection of $\sim 900 \mathrm{~s}$ in TQ magnets.

\section{SUMMARY}

The magnetic field measurements were performed in three TQ model magnets based on $\mathrm{Nb}_{3} \mathrm{Sn}$ conductor. The comparison with the results achieved in the short HGQ models and final MQXB magnets used in LHC IR region shows that the geometrical harmonics in TQ models are comparable with those in HGQ magnets within the measurement uncertainties. The HGQ production technology was subsequently improved and MQXB magnets demonstrated better geometrical harmonics. There is a possibility that further development of the $\mathrm{Nb}_{3} \mathrm{Sn}$ technology will lead to similar improvement of the field quality in the $\mathrm{Nb}_{3} \mathrm{Sn}$ quadrupoles.

The eddy current effects were substantially different in all three TQ magnets that is likely related to different interstrand contact resistances. This is consistent with the different mechanical prestresses in TQC01 and TQS01 models and also with different conductors in TQS01 and TQS02.

The long-term decay and snap-back effects were not observed in either of the TQ models that is different from the results of HGQ and MQXB magnets that demonstrated prominent decay and snap-back. However, it is consistent with the absence of snap-back in other $\mathrm{Nb}_{3} \mathrm{Sn}$ magnets made of similar conductors.

\section{REFERENCES}

S. A. Gourlay et al., "Magnet R\&D for the US LHC accelerator research program,” IEEE Trans. Appl. Supercond., vol. 16, no.2, pp. 324-327, June 2006.

[2] G.V. Velev et al., "Magnetic Field Measurements of LHC Inner Triplet Quadrupoles Fabricated at Fermilab”, IEEE Trans. Appl. Supercond., vol. 17, 2007, pp. 1109-1112.

A.Yamamoto et al., "Production and measurement of the MQXA series of LHC low- $\beta$ insertion quadrupoles", IEEE Trans. Appl. Supercond. vol. 15, 2005, pp. 1084-1089.

[4] S. Feher et al., Development and Test of LARP Technological Quadrupole (TQC) Magnet, IEEE Transactions on Applied Superconductivity, Vol. 17, No. 2, June 2007, pp.1126-1129.
Formatted: Bullets and Numbering

Formatted: Bullets and Numbering

Formatted: Bullets and Numbering 
[5] S. Caspi et al., Fabrication and Test of TQS01-A $90 \mathrm{~mm}$ Nb3Sn Quadrupole Magnet for LARP, IEEE Transactions on Applied Superconductivity, Vol. 17, No. 2, June 2007, pp.1122-1125.

[6] G.V. Velev et al., "Field quality measurements of the LQXB inner triplet quadrupoles for LHC", IEEE Trans. Appl. Supercond., vol. 15, 2005, pp. 1102-1105

N. Andreev et al., "Field quality in Fermilab-built models of high gradient quadrupole magnets for the LHC interaction region”, IEEE

Formatted: Bullets and Numbering Trans. Appl. Supercond., vol. 10, 2000, pp. 107-110.

[1] A. Jain, "Harmonic coils", CERN 98-05, Aug. 1998.

[8] D. A. Finley et al., "Time dependent chromaticity changes in the Tevatron", in Proc. 1987 PAC., Washington, DC, 1987, pp. 151-153.

[9] G.V. Velev et al., "Measurements of the Persistent Current Decay and Snapback Effect in Tevatron Dipole", IEEE Trans. Appl. Supercond., vol. 17, 2007, pp. 1105-1108. 